\title{
Adherencia o deserción de adolescentes en psicoterapia por agresiones sexuales: la perspectiva de sus terapeutas
}

\author{
Adherence or Drop Out of Adolescents in Psychotherapy Due to \\ Sexual Abuse: their Therapist's Perspective
}

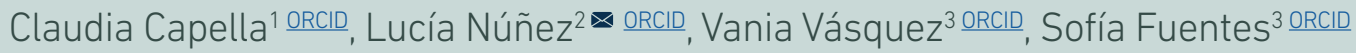

Universidad de Chile

Chile

Fecha correspondencia:

Recibido: noviembre 30 de 2019.

Aceptado: octubre 17 de 2020.

Forma de citar:

Capella, C., Núnez, L., Vásquez, V.,

\& Fuentes, S. (2020). Adherencia

0 deserción de adolescentes

en psicoterapia por agresiones

sexuales: la perspectiva de sus

terapeutas. Rev. CES Psico, 13(3),

124-141.

Open access

(c) Copyright

Licencia creative commons

Ética de publicaciones

Revisión por pares

Gestión por Open Journal System DOl: http://dx.doi.org/10.21615/

cesp.13.3.8

ISSN: 2011-3080

\section{Resumen}

Un alto porcentaje de adolescentes que asisten a psicoterapia por haber sido víctimas de agresiones sexuales desertan de su tratamiento, afectando así su proceso de superación. Existe escasa investigación respecto a qué precipita la deserción o favorece la adherencia en estos casos. Objetivo: describir características del sistema consultante y del proceso psicoterapéutico de adolescentes que han sido víctimas de agresiones sexuales que adhieren y que desertan de la psicoterapia, desde la perspectiva de sus psicoterapeutas, explorando sus similitudes y diferencias. Método: se realizaron entrevistas semiestructuradas a psicoterapeutas que atendieron 16 casos de adolescentes que han sido víctimas de agresiones sexuales, 8 que desertaron de la psicoterapia y 8 que adhirieron hasta el alta. Se realizó un análisis narrativo temático de las entrevistas. Resultados: las principales similitudes identificadas fueron contextos sociofamiliares multiproblemáticos, recursos personales en los adolescentes y una relación psicoterapéutica positiva. Las diferencias se enfocaron en los adultos responsables, quienes en los casos de deserción mostraban bajo apoyo, una relación más conflictiva con el adolescente, escaso involucramiento en la psicoterapia y una relación frágil con el psicoterapeuta. Los adolescentes que desertaron presentaron motivos de consulta no asociados con la agresión sexual y se caracterizaron por ser socialmente poco integrados, a diferencia de los que adhirieron. Discusión: los fenómenos de adherencia y deserción a psicoterapia son complejos y multifactoriales. Junto con factores asociados al sistema consultante, se destacan elementos del proceso psicoterapéutico que propician la adherencia, y que, por tanto, pueden ser abordados tempranamente para favorecer la adherencia a la psicoterapia.

Palabras clave: Deserción Psicoterapéutica, Psicoterapia, Adherencia, Adolescentes, Agresiones Sexuales, Psicoterapeutas.

Comparte

\section{Abstract}

A high percentage of adolescents who attend psychotherapy due to se- xual abuse drop out of treatment, affecting the overcoming. There is little research 
Sobre los autores:

1. Doctora en Psicología. Académica Departamento de Psicología Universidad de Chile. Investigadora Adjunta del Instituto Milenio para la Investigación en Depresión y Personalidad (MIDAP).

2. Doctora (c) en Psicoterapia. Pontificia Universidad Católica de Chile/Universidad de Chile. Miembro del Instituto Milenio para la Investigación en Depresión y Personalidad (MIDAP).

3. Psicóloga. Universidad de Chile. on what precipitates desertion or promotes adherence in these cases. Aim: describe features of the consulting system and the psychotherapeutic process of adolescents who have been sexually abused who adhere and drop out of psychotherapy, in order to explore similarities and differences from the perspective of their psychotherapists. Methods: Semi-structured interviews were conducted with psychotherapists who attended 16 cases of adolescent victims of sexual abuse, 8 who deserted from psychotherapy and 8 who continued until discharge. Thematic narrative analysis of the interviews was developed. Results: the main similarities described were multi-problematic social and family contexts, personal resources in the adolescents and a positive psychotherapeutic relationship. Differences were focused on caretakers, who in the drop out cases showed low support and a more conflictive relationship with the adolescent, little involvement in psychotherapy and a poor relationship with the psychotherapist. Adolescents who deserted had consultation purposes not related with sexual abuse and low social integration, unlike those who adhered. Discussion: the phenomena of adherence and dropout of psychotherapy are complex and multifactorial. Along with factors associated with the consulting system, elements of the psychotherapeutic process that support adherence stand out, which can be addressed early in the process.

Keywords: Psychotherapy Drop Out, Adherence, Adolescents, Sexual Abuse, Psychotherapists.

\section{Introducción}

Las agresiones sexuales hacia niños ${ }^{1}$ y adolescentes (en adelante ASI por Abuso Sexual Infantil) constituyen un problema con alta prevalencia (Pereda, Guilera, Forns, \& Gómez-Benito, 2009; Fondo de las Naciones Unidas para la Infancia (UNICEF), 2012). Se han descrito ampliamente las consecuencias psicológicas negativas en quienes han sido víctimas de ASI (Adam, Mrug, \& Knight, 2018; Guha, Luebbers, Papalia, \& Ogloff, 2019) y el beneficio de las intervenciones psicológicas y de salud mental en estos casos (Hetzel-Riggin, Brausch, \& Montgomery, 2007; Lippert, Favre, Alexander, \& Cross, 2008).

En Chile, el Servicio Nacional de Menores (SENAME, 2015) ofrece programas especializados en atención a niños y adolescentes que han sido víctimas de agresiones sexuales o maltrato grave. Estudios realizados con población infanto-juvenil que finalizaron exitosamente su psicoterapia en estos programas (Águila et al., 2016; Capella et al., 2016; Dussert et al., 2017), mostraron que la superación de la experiencia vivida se desarrolla a través de un proceso de cambio que implica bienestar, crecimiento, fortalecimiento y comprensión de la experiencia abusiva como parte de la vida. En este proceso son relevantes factores extraterapéuticos como el apoyo familiar, y psicoterapéuticos como sentirse comprendido durante la psicoterapia y un vínculo confiable y seguro con el terapeuta.

Estos estudios se realizaron con niños y adolescentes que finalizaron su psicoterapia, y que, por tanto, cumplieron con una condición básica: la adherencia al tratamiento (Deakin, Gastaud, \& Tiellet, 2012). La adherencia al tratamiento hace referencia al seguimiento por parte del paciente de las instrucciones entregadas por los profesionales de salud (Horne et al., 2005), es decir, la implicación del paciente en un tratamiento aceptado de mutuo acuerdo con el fin de producir el resultado terapéutico deseado (Di Matteo \& DiNicola, 1982). 
La deserción puede acarrear graves consecuencias como el deterioro de la situación de los pacientes y la obtención de resultados más pobres respecto a los que permanecen en tratamiento; además, puede incrementar los costos de la prestación, debido al gasto institucional y desaprovechamiento de recursos humanos en intervenciones inconclusas (Baruch, Vrouva, \& Fearon, 2009; Hernández, Acosta, \& Pereira, 2009; Luk et.al, 2001; Swift, Spencer, \& Goode, 2018).
Lo contrario de la adherencia al tratamiento se conoce con el concepto de deserción. En la población infanto-juvenil, la deserción del proceso psicoterapéutico presenta alta prevalencia, con tasas que varían entre $16 \%$ y $75 \%$, según un metaanálisis de estudios a nivel internacional realizado por De Haan, Boon, Hoeve, Jong y Vermeiren (2013). La deserción puede acarrear graves consecuencias como el deterioro de la situación de los pacientes y la obtención de resultados más pobres respecto a los que permanecen en tratamiento; además, puede incrementar los costos de la prestación, debido al gasto institucional y desaprovechamiento de recursos humanos en intervenciones inconclusas (Baruch, Vrouva, \& Fearon, 2009; Hernández, Acosta, \& Pereira, 2009; Luk et.al, 2001; Swift, Spencer, \& Goode, 2018).

Existen diversas definiciones de deserción (Deakin et al., 2012, Nock \& Ferriter, 2005; Rondón, Otarola, \& Salamanca, 2009) y según una de las más aceptadas, la deserción es la situación en la que el paciente termina tempranamente el tratamiento sin llegar a un acuerdo con el terapeuta (O'Keeffe, Martin, Target, \& Midgley, 2019): puede ocurrir en cualquier momento del proceso y el criterio clínico respecto a lo inapropiado de la finalización del tratamiento está acorde a los progresos del paciente (0’Keeffe et al., 2018). En la presente investigación la definición de deserción psicoterapéutica considera el criterio del psicoterapeuta, según la cual esta supone que los pacientes dan término a la psicoterapia sin lograr los objetivos establecidos en el contrato terapéutico (Deakin et al., 2012).

Tanto la adherencia como la deserción son fenómenos complejos y relacionados entre sí (Deakin et al., 2012); están determinados por múltiples factores (Hernández et al., 2009), relativos al paciente, su familia y la psicoterapia. Respecto al paciente, la mayoría de los estudios plantean que un nivel socioeconómico bajo se relaciona negativamente con la adherencia (Kazdin, Mazurick, \& Bass, 1993; Pelkonen, Marittunen, Laippala, \& Lönnqvist, 2000; Reis \& Brown, 1999), así como la pertenencia a un grupo étnico minoritario (Armbruster \& Schwab-Stone, 1994; Furnham \& Wong, 2007). También se ha asociado la deserción con la patología del paciente y la gravedad de sus síntomas (Bueno, Córdoba, Escolar, Carmona, \& Rodríguez, 2001; Hamberger, Lohr, \& Gottlieb, 2000; Killaspy, Banerjee, King, \& Lloyd, 2000; Matas, Staley, \& Griffin, 1992; Reich \& Berman, 2018), con las percepciones y creencias negativas de los usuarios respecto a los servicios de salud (Gearing, Schwalbe, \& Short, 2012) y con la presencia de deserciones previas (Marks, Lovell, Noshirvani, Livanou, \& Thrasher, 1998).

Se han planteado diversos modelos de factores de riesgo y protectores de la adherencia/deserción a tratamientos psicoterapéuticos (Kazdin, 1996); no obstante, la mayor parte de estos modelos y estudios se enfocan en las características de "entrada" a terapia del paciente y un menor número se centran en el proceso psicoterapéutico (Ross \& Werbart, 2013). En cuanto a los factores relativos a la psicoterapia, se ha encontrado que la tasa de deserción es mayor cuando los psicoterapeutas no logran precisar el modo cómo el paciente conceptualiza el problema (Epperson, Bushway, \& Warman, 1983; Wierzbicki \& Pekarik, 1993); mayor adherencia en pacientes que reciben terapia combinada (psicológica y farmacológica) (Edlund et al., 2002), y aquellos que perciben que sus psicoterapeutas tienen capacidad de escuchar, muestran una actitud flexible, inspiran confianza y atienden a sus necesidades emocionales y afectivas (Freire, 1990). La alianza psicoterapéutica (Ross \& Werbart, 2013) y la ocurrencia de cambios en la situación del paciente en las primeras fases del tratamiento, juegan un papel relevante en la adherencia a la psicoterapia (Fernández, Pérez, Gloger, \& Krause, 2015); así como la capacidad de reparar rupturas en la alianza (Swift et al., 
Pág 127

En un estudio realizado con adolescentes consultantes a servicios de salud mental en Chile se observó que éstos presentaban reticencia inicial para participar en la psicoterapia; y en los casos de adherencia se encontró mayor nivel de acuerdo entre adolescente y psicoterapeuta en cuanto a la valoración de la efectividad de la psicoterapia y las razones del término de esta, que en los casos de deserción (Mendoza, 2016).
2018). La demora para recibir la atención requerida, por otro lado, afecta negativamente la adherencia (Hillis, Eagles, \& Eagles, 1993).

Aun cuando la mayor parte de la literatura científica sobre deserción y adherencia a la psicoterapia proviene de estudios realizados con adultos, existen algunos con población infanto-juvenil; no obstante, la mayoría de éstos desconoce distinciones en función de la edad e investigan en conjunto niños y adolescentes (Viale-Val, Rosenthal, Curtis, \& Marohn, 1984); además, se han centrado en la no adherencia al tratamiento en su etapa inicial (Demarest, Heger, Foy, \& Leskin, 1996; Fernandez et al., 2015; Lippert et al., 2008).

Específicamente, la deserción en población infanto-juvenil se ha asociado a la presencia de factores familiares tales como limitaciones financieras y de acceso a la atención en salud mental (Edlund et al., 2002), necesidades de cuidado de los niños, patología en los padres (Pellerin, Costa, Weems, \& Dalton, 2010), falta de participación de la familia y desestimación por parte de los padres de la necesidad de intervención psicoterapéutica (Edlund et al., 2002); o desacuerdo entre padres y psicoterapeutas con respecto al diagnóstico y los resultados buscados (Gearing et al., 2012). A esto se suma que, una de las preocupaciones de los padres es el estigma que podría recaer en sus hijos por participar en una psicoterapia (Gearing et al., 2012).

Estudios recientes con adolescentes con depresión evidencian una alta proporción de deserción a la psicoterapia (alrededor del 40\%), situación que obedece más a aspectos del tratamiento como, por ejemplo, sesiones perdidas y una relación psicoterapéutica inicial pobre, que a factores pretratamiento relacionados con la familia y el paciente (0’Keeffe et al., 2018; 2019). Los adolescentes explican su deserción con argumentos como la falta de utilidad de la psicoterapia, presentar mejoría o dificultades que les impiden asistir (0'Keefee et al., 2019).

En un estudio realizado con adolescentes consultantes a servicios de salud mental en Chile se observó que éstos presentaban reticencia inicial para participar en la psicoterapia; y en los casos de adherencia se encontró mayor nivel de acuerdo entre adolescente y psicoterapeuta en cuanto a la valoración de la efectividad de la psicoterapia y las razones del término de esta, que en los casos de deserción (Mendoza, 2016).

Estudios sobre deserción en psicoterapia en niños y adolescentes que han sido víctimas de trauma y ASI son escasos; los existentes son mayoritariamente cuantitativos, realizados en países anglosajones y centrados en variables demográficas. Según sus hallazgos, en niños y adolescentes que asisten a psicoterapia por experiencias de ASI, la no adherencia se asocia con padres más jóvenes, menor nivel socioeconómico, pertenencia a grupos minoritarios, niños con síntomas externalizantes y de estrés postraumático, así como mayor número de experiencias traumáticas y negligencia parental (Lippert et al., 2008).

Por otro lado, se ha reportado que los niños de menor edad con experiencias traumáticas culminan el tratamiento en mayor proporción que los adolescentes (Eslinger, Sprang, \& Otis, 2014; Ormhaug \& Jensen, 2018; Wamser-Nanney \& Steinzor, 2017). La baja participación parental en la primera sesión y la percepción de que los padres no aprueban el tratamiento también se asocia a mayor deserción (Ormhaug \& Jensen, 2018), siendo también la participación de los cuidadores en el tratamiento asociado a ASI una variable esencial en la terminación o no del tratamiento (McPherson, Scribano, \& Stevens, 2012). 
Pág 128

El objetivo del presente estudio es describir las características del sistema consultante (adolescente y su familia) y del proceso psicoterapéutico de adolescentes que han sido víctimas de agresiones sexuales que adhieren y que desertan de la psicoterapia, desde la perspectiva de sus psicoterapeutas, explorando sus similitudes y diferencias.
Un estudio cualitativo realizado en Chile con adolescentes que han sido víctimas de ASI (Fuentes \& Vásquez, 2017; Núñez, Fuentes, Vásquez, \& Capella, 2016), señala que la deserción a la psicoterapia es un fenómeno multicausal. Los principales factores identificados se asocian con contextos sociales y familiares multiproblemáticos, escasa credibilidad y protección al adolescente frente a la ASI por parte del adulto responsable y, en menor medida, factores relativos a la psicoterapia y el centro de atención, como la dificultad de generar un motivo de consulta consensuado asociado al abuso, una relación psicoterapéutica deficiente con el adulto responsable e interrupción de las atenciones.

Teniendo en cuenta lo anterior, se advierte la necesidad de profundizar en las diferencias y similitudes entre los casos de deserción y de adherencia a la psicoterapia de pacientes adolescentes que han sido víctimas de ASI. En consecuencia, el objetivo del presente estudio es describir las características del sistema consultante (adolescente y su familia) y del proceso psicoterapéutico de adolescentes que han sido víctimas de agresiones sexuales que adhieren y que desertan de la psicoterapia, desde la perspectiva de sus psicoterapeutas, explorando sus similitudes y diferencias. Observar características comunes y aspectos diferenciales busca aportar a la comprensión del fenómeno y al diseño de lineamientos y estrategias que favorezcan la adherencia al tratamiento.

El presente estudio se centra en la perspectiva de los psicoterapeutas dada su calidad de principal interventor en el caso (Águila et al., 2016). Si bien el punto de vista del terapeuta ha sido escasamente estudiado (Jo \& Almao, 2002), su posición en el proceso psicoterapéutico le permite una visión más amplia respecto al funcionamiento psicológico del paciente, los distintos momentos de la psicoterapia y los procesos psicológicos que ahí se enmarcan; por lo cual proporciona una vasta comprensión del fenómeno (Altimir et al., 2010).

\section{Metodología}

Este estudio es de carácter cualitativo, se enfoca en comprender los fenómenos desde los marcos de referencia de las personas involucradas (Valles, 2003); en este caso, comprender desde la subjetividad de los psicoterapeutas, la adherencia o deserción de los adolescentes en psicoterapia por agresiones sexuales. El enfoque es narrativo, puesto que busca comprender los relatos que realizan los participantes de sus experiencias (Riessman, 2008), en este caso, el de los psicoterapeutas respecto al proceso psicoterapéutico llevado a cabo con adolescentes que adhieren o desertan de psicoterapia.

El presente estudio forma parte del proyecto "Cambio psicoterapéutico en agresiones sexuales: perspectiva de los niños/as y adolescentes que han sido víctimas, sus padres y sus psicoterapeutas" (FONDECYT N¹1140049). De las personas que conformaron la muestra de dicho proyecto, $25 \%$ desertaron $(\mathrm{N}=10)$ de la psicoterapia, y de este porcentaje, $90 \%$ eran adolescentes $(\mathrm{N}=9)$, frente a lo cual nace el interés por realizar el presente estudio. Los participantes del presente estudio se seleccionaron a través de un muestreo intencionado de los participantes del proyecto.

Para acceder a la muestra se contó con la colaboración de seis Centros de reparación en maltrato y abuso de la ciudad de Santiago, Chile. Estos Centros siguen lineamientos establecidos por el SENAME (2015), a través de un modelo de intervención psicosocial, en el que el/la psicólogo/a realiza psicoterapia con niños/adolescentes 
Pág 129

Como técnica de producción de los datos se utilizó la entrevista en profundidad (Valles, 2003), para acceder a la perspectiva de los terapeutas acerca del proceso. Se utilizó un guion temático de entrevista cuyos principales ejes fueron: expectativas, motivo de consulta, comprensión de la problemática, proceso y contenido del cambio, proceso y relación psicoterapéutica y cierre de la intervención. y el/la trabajador/a social realiza la intervención familiar. La totalidad de los casos incluidos fueron derivados por ASI desde el sistema judicial.

El proyecto macro recibió aprobación del Comité de Ética de la investigación de la Facultad de Ciencias Sociales de la Universidad de Chile. Tras recibir la autorización de los directivos de los Centros, se contactaron los consultantes que estuvieran iniciando psicoterapia y se invitaron a formar parte del estudio. Luego de la aceptación a participar y la firma de asentimientos y consentimientos informados por parte de los niños, adolescentes, sus padres y terapeutas, respectivamente, se registraron datos de los casos y se aplicaron instrumentos que evalúan aspectos sintomatológicos ${ }^{2}$ al inicio de la psicoterapia. Posteriormente, se realizaron entrevistas en diferentes momentos de la intervención (aproximadamente a los 6 meses, a los 12 y al final de la intervención). El presente estudio se centra en las entrevistas realizadas a los psicoterapeutas al finalizar el proceso, tanto en los casos de deserción como de adherencia.

Participaron en el presente estudio 13 psicoterapeutas ( 5 hombres, 8 mujeres; entre los 25 y 46 años, con diferentes enfoques teóricos), quienes atendieron en total 16 adolescentes (tres de los psicoterapeutas atendieron dos casos cada uno). Para favorecer la comprensión de los datos se hará referencia al total de 16 casos. Los adolescentes ${ }^{3}$ atendidos por psicoterapeutas tenían entre 12 y 17 años al inicio de la psicoterapia (edad promedio 14 años), 8 de los cuales desertaron de terapia y 8 adhirieron hasta el alta. Las psicoterapias duraron entre 3 y 9 meses (promedio 6 meses) en los casos de deserción, y entre 16 y 20 meses (promedio 18 meses) en los casos de adherencia. La madre fue el adulto responsable en la mayor parte de los casos. En su mayoría, la agresión sexual fue intrafamiliar, reiterada o crónica. En cinco de los casos de adherencia hubo cambios de psicoterapeuta durante el proceso, pero se hará referencia a las características del psicoterapeuta que participó de la entrevista considerada en este estudio. Las características muestrales se sintetizan en la tabla $1^{4}$.

Como técnica de producción de los datos se utilizó la entrevista en profundidad (Valles, 2003), para acceder a la perspectiva de los terapeutas acerca del proceso. Se utilizó un guion temático de entrevista cuyos principales ejes fueron: expectativas, motivo de consulta, comprensión de la problemática, proceso y contenido del cambio, proceso y relación psicoterapéutica y cierre de la intervención. Las entrevistas fueron realizadas por miembros del equipo de investigación en cada centro dentro del mes posterior al cierre del caso.

La técnica de análisis de información utilizada corresponde al análisis narrativo temático, cuya característica distintiva es centrarse en el contenido de los relatos que las personas construyen como formas básicas para otorgar sentido a sus vivencias (Riessman, 2008). Inicialmente se identificó la narrativa principal de la entrevista, y luego los contenidos específicos, que se organizaron en ejes temáticos y se agruparon en distintas unidades de análisis, para luego ser incluidos en unidades temáticas de mayor nivel de complejidad, según los objetivos de esta investigación, que utilizó como apoyo el programa Atlas ti versión 7.1 para el análisis de datos. 
Tabla 1. Características de la muestra

\begin{tabular}{|c|c|c|c|c|c|}
\hline & & & Deserción & Adherencia & Total \\
\hline \multirow{4}{*}{ Paciente } & \multirow{2}{*}{ Género } & Hombre & 2 & 3 & 5 \\
\hline & & Mujer & 6 & 5 & 11 \\
\hline & \multirow{2}{*}{ Edad } & 12-14 años & 2 & 6 & 8 \\
\hline & & 15-17 años & 6 & 2 & 8 \\
\hline \multirow{6}{*}{ Agresión } & \multirow{2}{*}{ Agresor } & Intrafamiliar & 5 & 6 & 11 \\
\hline & & Extrafamiliar conocido & 3 & 2 & 5 \\
\hline & \multirow{2}{*}{ Tipo de agresión } & Abuso sexual & 6 & 5 & 11 \\
\hline & & Violación & 2 & 3 & 5 \\
\hline & \multirow{2}{*}{ Cronicidad } & Única & 1 & 4 & 5 \\
\hline & & Repetida o crónica & 7 & 4 & 11 \\
\hline \multirow{3}{*}{$\begin{array}{l}\text { Adulto } \\
\text { responsable }\end{array}$} & \multirow{3}{*}{ Relación } & Madre & 4 & 6 & 10 \\
\hline & & Padre & 2 & 0 & 2 \\
\hline & & $\begin{array}{l}\text { Otra figura familiar } \\
\text { femenina }\end{array}$ & 2 & 2 & 4 \\
\hline \multirow{10}{*}{ Terapeuta } & \multirow{2}{*}{ Sexo } & Hombre & 3 & 3 & 6 \\
\hline & & Mujer & 5 & 5 & 10 \\
\hline & \multirow{4}{*}{ Enfoque } & Psicoanálisis & 5 & 1 & 6 \\
\hline & & Constructivista & 0 & 4 & 4 \\
\hline & & Sistémico & 1 & 3 & 4 \\
\hline & & Integrativo & 2 & 0 & 2 \\
\hline & \multirow{2}{*}{ Edad } & 25-34 años & 4 & 8 & 12 \\
\hline & & 35-46 años & 4 & 0 & 4 \\
\hline & \multirow{2}{*}{$\begin{array}{l}\text { Experiencia } \\
\text { clínica en AS }\end{array}$} & 1-5 años & 2 & 7 & 9 \\
\hline & & 5-15 años & 6 & 1 & 7 \\
\hline
\end{tabular}

Para guiar el análisis se seleccionaron las temáticas identificadas en casos de deserción en el trabajo previo realizado por Fuentes y Vásquez (2017), y Núñez, Fuentes, Vásquez y Capella $(2016)^{5}$, que corresponden a características del caso (familia, adulto responsable, adolescente), aspectos de reacción frente a la ASI, factores del proceso de intervención y relación psicoterapéutica. Estas temáticas fueron contrastadas mediante un análisis inter-caso entre los casos de deserción y de adherencia, y reorganizadas en los subtemas descritos en los resultados. En el proceso de análisis se utilizó la estrategia de triangulación, que valida los análisis a partir del acuerdo entre investigadores (Cornejo \& Salas, 2011).

\section{Resultados}

A partir del análisis de las entrevistas se organizaron los diferentes subtemas en dos unidades temáticas: a) el sistema consultante, que se refiere a características del adolescente y su familia, y las consecuencias frente a la ASI y, b) el proceso psicoterapéutico, que se relaciona con las características del proceso en términos de motivo de consulta y relación psicoterapéutica, entre otros. Se describirán características comunes y diferenciales entre los casos de deserción y adherencia en cada una de las unidades temáticas. 
Los psicoterapeutas describieron a los adolescentes de ambos grupos como activos, resilientes y con múltiples recursos personales; y señalaron que estas características favorecieron el proceso psicoterapéutico. Además, refirieron que los adolescentes buscaban ser escuchados y contenidos, es decir, presentaban gran necesidad del espacio psicoterapéutico.

\section{Características del sistema consultante}

Contexto sociofamiliar

Los psicoterapeutas describieron en los casos de deserción y de adherencia contextos sociofamiliares con múltiples problemáticas, tales como hacinamiento, violencia intrafamiliar y consumo de drogas. Además, identificaron aspectos diferentes entre los casos: algunos de los casos de adherencia presentaron antecedentes de otros tipos de maltrato además de la ASI, tales como abandono o maltrato físico, lo cual no fue referido en los de deserción.

En los casos de deserción se describieron dinámicas familiares complejas, caracterizadas por el hermetismo y silenciamiento frente a los conflictos. De acuerdo con los terapeutas, este tipo de funcionamiento dificultó la intervención, al instalarse una estrategia evitativa que rehúye el abordaje y la elaboración del abuso.

\section{Adulto responsable y su relación con el/la adolescente}

Los terapeutas presentaron visiones diferentes de los adultos responsables según los casos de adherencia y deserción. En los de adherencia, si bien primó una visión más positiva de esta figura que en los de deserción, se apreciaron dos descripciones diferentes: por un lado, adultos responsables con limitaciones para visualizar al adolescente dado que estaban inmersos en conflictos familiares, y aunque presentaban dificultades en la relación con el adolescente al inicio de la psicoterapia, mostraron interés en mejorarla. Se apreció un avance en esta relación a lo largo de la psicoterapia en tanto el adulto responsable participó del proceso y acompañó al adolescente. La segunda descripción se refiere a adultos responsables identificados como figuras de apoyo y protectoras de los adolescentes desde el inicio, con una buena relación entre ambos, lo cual se consideró un factor favorecedor del proceso.

"Yo creo que la mamá siempre fue muy protectora, siempre estaba muy muy preocupada de que ella viniera, eh ... también no solamente acá, sino que ella se moviliza, eh... ella se movilizaba mucho en llevarla a las diferentes redes donde ella tenía que concurrir" (Juan, adherenciab).

El comportamiento de los adultos responsables de los adolescentes en los casos de deserción se describió como inadecuado, egocéntrico y con dificultades para visualizar las necesidades de sus hijos/as, a quienes culpaban por sus problemas. La relación entre ambos se caracterizó como conflictiva y distante, y los psicoterapeutas observaron desconfianza mutua, constantes disputas y escasa comunicación. No lograron configurarse como figuras protectoras para el adolescente, tanto previa como posteriormente a la agresión sexual, y, en algunos casos, fueron descritos como maltratadores del adolescente.

\section{Adolescentes}

Los psicoterapeutas describieron a los adolescentes de ambos grupos como activos, resilientes y con múltiples recursos personales; y señalaron que estas características favorecieron el proceso psicoterapéutico. Además, refirieron que los adolescentes buscaban ser escuchados y contenidos, es decir, presentaban gran necesidad del espacio psicoterapéutico. 
Pág 132

En los casos de adherencia, el adulto responsable del adolescente daba credibilidad a la agresión sexual y se constituyó en una figura protectora, aun cuando no hubiera credibilidad de otros miembros de la familia.
"Los recursos del adolescente al ser él bien reflexivo, eh, al tener también como un mundo emocional bien rico (...), de la capacidad de vincularse, de la capacidad de poder reconocer los espacios de ayuda, yo creo que principalmente los recursos del adolescente (...) favorecieron harto" (Bárbara, adherencia).

Los psicoterapeutas refirieron que, en los casos de adherencia, los adolescentes participaban en diferentes actividades sociales y tenían relaciones cercanas con sus pares (amigos, novio/a); redes de apoyo que actuaron como factores protectores de sus procesos psicoterapéuticos. En cambio, en los casos de deserción, los psicoterapeutas advirtieron menor integración social de los adolescentes, esto es, tendían a ser más solitarios.

Significación y consecuencias de la agresión sexual a nivel individual y familiar Este aspecto presentó importantes diferencias desde la visión de los psicoterapeutas. En los casos de adherencia, el adulto responsable del adolescente daba credibilidad a la agresión sexual y se constituyó en una figura protectora, aun cuando no hubiera credibilidad de otros miembros de la familia. No obstante, en algunos casos se presentaban problemáticas familiares derivadas de la agresión sexual, como la afectación emocional del adulto responsable, rupturas entre los miembros de la familia que daban credibilidad o no, y preocupación por el contacto de hermanos menores con el agresor.

En los casos de deserción, los psicoterapeutas observaron ausencia o ambivalencia en la credibilidad de la familia y el adulto responsable frente a la situación de abuso, $y$, en consecuencia, presentaban una postura pasiva o poco protectora; condición identificada como obstaculizadora del proceso psicoterapéutico. En algunos casos el adulto responsable no denunció la ASI al sistema judicial y la denuncia se presentó desde otros contextos.

Los psicoterapeutas reportaron que, en los casos de adherencia, los adolescentes presentaban sintomatología inicial aguda y reactiva a la agresión sexual, de tipo depresiva, además, autolesiones y/o intentos suicidas y dificultades relacionales. Estos adolescentes significaron la agresión sexual como una vivencia traumática, salvo en dos casos que contaban con procesos psicoterapéuticos previos.

Respecto a la significación otorgada a la agresión sexual, los psicoterapeutas señalaron que, en casi todos los casos de deserción, los adolescentes la consideraban no traumática y no asociaban sus dificultades con la misma. Sin embargo, los psicoterapeutas reportaron diversas consecuencias en el desarrollo psicosexual de los adolescentes en la mayoría de los casos, tales como dificultades para relacionarse con pares, establecimiento de vínculos erotizados o descontrol en el ámbito de la sexualidad.

"Él (paciente) dice que la, como la sexualización: que él, a él le angustia un poco, como... él decía que se excitaba mucho (...) no? como que eso le generaba mucha angustia, como descontrol también (...) él no tenía elementos para regular, lo que él iba a hacer era como satisfacer, y en eso en el encuentro con el otro se... se desajustaba (...)" (Javier, deserción). 
Tanto en los casos de adherencia como en los de deserción, los psicoterapeutas describieron una buena relación psicoterapéutica con el adolescente que favoreció el proceso y operó como "enganche" hacia la psicoterapia. Caracterizaron esta relación como cercana, afable y de confianza, lo que facilitó la apertura del paciente, quien gradualmente confiaba contenidos significativos al psicoterapeuta y se sentía acogido y validado por este, al tiempo que se apropiaba del espacio.

\section{Características del proceso psicoterapéutico}

Motivo de consulta y motivación hacia la psicoterapia

En los casos de adherencia el motivo de consulta central de los adolescentes y su adulto responsable fue la ASI. En general, los adolescentes manifestaron alta motivación hacia la psicoterapia; y, en algunos casos no, debido al temor de abordar la experiencia abusiva, sin embargo, la motivación aumentó al trabajar gradualmente respetando los tiempos del paciente.

Del mismo modo, en los casos de adherencia, los adultos responsables presentaron alta motivación por asistir a psicoterapia, se comprometieron y participaron tanto como "padres", al respaldar el proceso de sus hijos/as, como "pacientes", al recibir apoyo directo desde los Centros.

Por otro lado, en varios casos de deserción, la ASI no fue el motivo de consulta principal de los adolescentes. Los psicoterapeutas observaron en los adolescentes que desertaron una necesidad del espacio psicoterapéutico, como un lugar de escucha, desahogo y contención respecto de otros conflictos; lo que se reflejaba en una alta motivación por asistir a psicoterapia.

"Yo creo que pudo aparecer algo de ella (paciente), de su rabia, de su... conflicto con la madre... y eso generó interés de asistir y de sentirse como... de tener un espacio eh, distinto, y poder hablar quizás, y expresar emociones que... que, no tengo muy claro si en otro lugar las podía expresar" (Josefa, deserción)

Sin embargo, para los psicoterapeutas, en los casos de deserción, los adultos responsables no lograron visualizar la necesidad de la psicoterapia para su hijo/a, y su motivo de consulta era inexistente, ajeno a la situación de ASI o se relacionaba con una queja hacia el adolescente y estaba enfocado en modificar su conducta.

"Cuando ella llega (madre del paciente) yo creo que la motivación es inercial, que quiero decir, que a ella le dijeron que era importante que hiciera el adolescente un proceso terapéutico, pero ella no tiene un convencimiento afectivo de digamos, de eh, de que eso pudiera ser relevante para su hijo" (Carlos, deserción)

Consecuentemente, los adultos responsables presentaron baja participación, compromiso emocional y apoyo al proceso del adolescente, resistencias de difícil manejo y en algunos casos desestimaron la intervención.

"La mamá vino a depositar, cámbienmela y, mm, y como no cambiaba la chica (risa) la mamá probablemente desestimó este lugar (...) aparte que se lo decía la mamá (a la adolescente): '¡Y tú que estás yendo al psicólogo!, jno te sirve de nada!, ¡no has cambiado nada!'” (Fabiola, deserción)

Relación psicoterapéutica con el adolescente

Tanto en los casos de adherencia como en los de deserción, los psicoterapeutas describieron una buena relación psicoterapéutica con el adolescente que favoreció el proceso y operó como "enganche" hacia la psicoterapia. Caracterizaron esta relación como cercana, afable y de confianza, lo que facilitó la apertura del paciente, quien gradualmente confiaba contenidos significativos al psicoterapeuta y se sentía acogido y validado por este, al tiempo que se apropiaba del espacio. El psicoterapeuta se posicionaba en un rol de escucha, apoyo y respeto por los tiempos de los pacientes 
Pág 134

En los casos de deserción, los psicoterapeutas mencionaron que los adultos responsables actuaron como los principales obstaculizadores, dado que no se comprometieron ni se instalaron en el tratamiento, más bien lo desestimaron, al punto de que un padre prohibió la asistencia del adolescente a la psicoterapia.
En los casos de deserción, junto con sentimientos positivos hacia los adolescentes, los psicoterapeutas percibieron un impacto emocional particular, relacionado con su preocupación y necesidad de protegerlos frente a situaciones de desprotección y abandono familiar en las que los visualizaban. Esto se acompañaba de expectativas de que los adolescentes pudieran independizarse de sus familias y sostenerse con sus propios recursos, y cuando no era posible, les generaba frustración.

\section{Relación entre psicoterapeuta y adulto responsable}

La relación del psicoterapeuta con el adulto responsable tendió a ser positiva en los casos de adherencia y negativa en los de deserción. En estos últimos, los psicoterapeutas reportaron sentimientos negativos y una visión crítica hacia los adultos responsables, por no visualizar las necesidades del adolescente y protegerlo. Por tanto, describieron las relaciones como distantes, tensas y sin confianza.

"Fue difícil con la madre, la madre cualquier cosa que uno dijera o no dijeras, ella se sentía atacada como madre, emm, entonces estaba muy a la defensiva, no, creo que no se logró ninguna relación con ella" (Fabiola, deserción)

\section{Curso y cierre del proceso psicoterapéutico}

Los casos de adherencia y deserción presentaron claras diferencias al constituir dos trayectorias opuestas respecto del curso del tratamiento.

En general, en los casos de adherencia el cierre se relacionó con el cumplimiento de los objetivos de la intervención. Dentro de los logros psicoterapéuticos, los psicoterapeutas destacaron la disminución de síntomas, la elaboración de las secuelas traumáticas de la ASI, una mejor relación con el adulto responsable y el fortalecimiento de habilidades sociales del adolescente.

En algunos casos de adherencia el proceso psicoterapéutico enfrentó obstáculos respecto a su continuidad debido principalmente a la rotación de los psicoterapeutas; no obstante, fue posible sostener la intervención dada la relación con el trabajador social que conformaba la dupla profesional. Otros elementos que amenazaron la continuidad del proceso fueron la asistencia irregular de algunos pacientes, y el cumplimiento de tiempos de intervención dispuestos por los lineamientos de los Centros.

En los casos de deserción, los psicoterapeutas mencionaron que los adultos responsables actuaron como los principales obstaculizadores, dado que no se comprometieron ni se instalaron en el tratamiento, más bien lo desestimaron, al punto de que un padre prohibió la asistencia del adolescente a la psicoterapia. Varios adultos responsables desertaron primero, y a pesar de la alta motivación de los adolescentes, estos no lograron continuar por sí solos.

"Creo que efectivamente la madre no se instaló, por lo tanto, la chica hizo el esfuerzo de instalarse, eso es cierto, pero no hubo persistencia de nadie (...) la chica lo intentó porque vino las tres o cuatro últimas veces sola, entonces se notaba que ella necesitaba el espacio, porque ella venía" (Fabiola, deserción)

De este modo, se interrumpieron los procesos de modo abrupto y en ninguno de ellos fue posible realizar un cierre, pese a los intentos de los profesionales del Centro por retomar. En algunos casos de deserción los psicoterapeutas añadieron que el funcionamiento institucional precipitó el proceso de abandono, al generar periodos de interrupción de las atenciones por asuntos administrativos. 
Pág 135

Los psicoterapeutas identifican elementos favorecedores y obstaculizadores del proceso psicoterapéutico, mientras en los casos de deserción dominan los que entorpecen el proceso, tales como, una familia hermética y poco protectora, o un adulto responsable con escaso compromiso con la terapia; en los casos de adherencia prevalecen los aspectos favorecedores, tales como la presencia de un adulto responsable protector y un adolescente con relaciones sociales en las que encuentra apoyo.
Respecto al cumplimiento de objetivos psicoterapéuticos, en los casos de deserción, los psicoterapeutas señalaron que solo se alcanzaron algunos objetivos iniciales, como la disminución de síntomas y la sensación de bienestar del adolescente al contar con el espacio psicoterapéutico. La elaboración de la experiencia de abuso no se logró.

Finalmente, en los casos de deserción los psicoterapeutas reportaron sentimientos antagónicos; por un lado, empatía, compromiso y deseo de proteger al adolescente y, por otro, molestia y crítica hacia el adulto responsable, junto con la frustración asociada a la deserción del caso. Así, los psicoterapeutas mencionaron la necesidad de mayor supervisión clínica e indicaron que esta podría mejorar su intervención en dichos procesos.

\section{Discusión y conclusión}

Los resultados de este estudio permiten concluir que, tanto la deserción como la adherencia de adolescentes que asisten a psicoterapia por ASI, son entendidas por los psicoterapeutas como procesos complejos y multifactoriales, influidos por elementos del sistema consultante y de la psicoterapia; lo que coincide con otros estudios (Kazdin, 1996; Hernández et al., 2009).

Las principales similitudes identificadas por los psicoterapeutas tanto en los casos de deserción como los de adherencia son contextos sociofamiliares desfavorables, en los cuales la credibilidad respecto al ASI es compleja, sea porque no existe o es ambivalente, como se ha descrito previamente (Centro de Asistencia a Víctimas de Atentados Sexuales [CAVAS], 2011). Otro elemento común es la visualización de los adolescentes como poseedores de diversos recursos y su motivación con la psicoterapia, lo que genera una relación psicoterapéutica positiva.

Así mismo, los psicoterapeutas identifican elementos favorecedores y obstaculizadores del proceso psicoterapéutico, mientras en los casos de deserción dominan los que entorpecen el proceso, tales como, una familia hermética y poco protectora, o un adulto responsable con escaso compromiso con la terapia; en los casos de adherencia prevalecen los aspectos favorecedores, tales como la presencia de un adulto responsable protector y un adolescente con relaciones sociales en las que encuentra apoyo.

En cuanto a los adultos responsables, los psicoterapeutas describen, en los casos de deserción relaciones conflictivas entre estos adultos y los adolescentes, en las que son frecuentes la falta de apoyo y protección frente a la situación de ASI; sumado a una baja o nula motivación de los adultos responsables hacia la psicoterapia, que imposibilita una relación psicoterapéutica positiva que favorezca la adherencia. Esto difiere en los casos de adherencia, en los que los psicoterapeutas observan apoyo al adolescente, motivación y participación en el proceso psicoterapéutico y una buena relación con el psicoterapeuta por parte del adulto responsable.

Mientras algunos estudios no mencionan la relevancia de los adultos responsables en la deserción de adolescentes a la psicoterapia (0'Keeffe et al., 2019), otros, realizados con víctimas de trauma, refieren la importancia del apoyo parental en tratamientos emocionalmente demandantes (Ormhaug \& Jensen, 2018); señalan la necesidad de una mirada sistémica de la psicoterapia con adolescentes en casos de trauma y ASI, que adopte estrategias de compromiso y enganche con la familia y, en especial con los adultos responsables (McPherson et al., 2012; Saxe, Ellis, Fogler, \& 
Pág 136

En muchos casos los factores pretratamiento más que los factores del tratamiento explican la deserción (0’ Keefee et. al 2018), es relevante detectar el modo como factores previos al tratamiento, por ejemplo, la significación y reacción del adolescente y su familia ante la agresión sexual (ASI), se relacionan con factores de la psicoterapia, como el motivo de consulta y la motivación al mismo.
Navalta, 2012). Es crucial involucrar activa y tempranamente a los padres en la psicoterapia como actores esenciales en el proceso y trabajar con ellos las diferencias en el motivo de consulta o las dificultades con sus hijos (Ormhaug \& Jensen, 2018), y de este modo favorecer la adherencia. Por lo consiguiente, y pese al deseo de algunos psicoterapeutas de que los adolescentes sostengan autónomamente sus procesos, es importante, que los terapeutas fortalezcan una mirada evolutiva de la situación, ya que la agencia de los adolescentes aún se sostiene en la relación y el apoyo familiar (Sepúlveda, 2013).

Otra diferencia que sobresale entre los casos de adherencia y deserción es la comprensión de la agresión sexual por parte de los adolescentes y sus padres o adultos responsables. La ASI es escasamente problematizada o planteada como motivo de consulta por los adolescentes en los casos de deserción, al contrario, se presentan motivos de consulta que no están directamente relacionados con la ASI. En cambio, en los casos de adherencia hay una problematización de la ASI, síntomas reactivos en el adolescente y un motivo de consulta vinculado a la misma. Finalmente, el curso de la psicoterapia implica que esta temática es abordada y se observan logros. Esto se entiende en la medida que la experiencia de ASI no necesariamente debe comprenderse como traumática por sí misma, depende, más bien, de la significación que le otorgue cada persona (Capella, 2017). Si la significación de la ASI no es traumática, es posible que el ingreso a un centro especializado en ASI pueda no ser relevante para el adolescente o su familia. 0 bien, pudiera ser que, como consecuencia de una significación traumática, el adolescente evite abordar la temática. Al respecto, Gearing et al. (2012) señalan que el desacuerdo entre padres y terapeutas con respecto al diagnóstico y los resultados buscados con la psicoterapia podría ser un factor asociado con la deserción. Por ende, como implicancia en términos clínicos resulta relevante trabajar inicialmente en construir el motivo de la consulta y la motivación a la psicoterapia con la familia y el adolescente, especialmente en el abordaje de la ASI, si se considera pertinente dadas sus consecuencias traumáticas; o bien evaluar en cada caso la pertinencia de la atención en un centro especializado en ASI, la necesidad de otras intervenciones o de coordinar con otras redes para responder a otras demandas.

Teniendo en cuenta que, en muchos casos los factores pretratamiento más que los factores del tratamiento explican la deserción (0’Keefee et. al 2018), es relevante detectar el modo como factores previos al tratamiento, por ejemplo, la significación y reacción del adolescente y su familia ante la ASI, se relacionan con factores de la psicoterapia, como el motivo de consulta y la motivación al mismo. Reconocer estos elementos al inicio de la psicoterapia permitirá identificar los casos que requieren más apoyo y trabajo, y de esta manera favorecer la adherencia y finalización de la intervención, y alcanzar los objetivos psicoterapéuticos (Eslinger et al., 2014; O’ Keefee et al., 2018).

Aun cuando en este estudio se identificaron elementos tanto del proceso psicoterapéutico como del sistema consultante que inciden en la deserción o adherencia en los casos de ASI, desde la perspectiva de los psicoterapeutas, los principales obstaculizadores del proceso psicoterapéutico se encuentran en el sistema consultante. Gearing et al. (2012) indican que esta situación puede obedecer a sesgos de atribución presentes en los clínicos, por los cuales, tienden a considerar sus acciones como promotoras de la adherencia y observan las principales barreras en los consultantes. En este sentido, una de las limitaciones de la presente investigación es que solo contempló la visión de los psicoterapeutas; por lo cual, se recomienda la realización 
de estudios que complementen esta perspectiva con la de los adolescentes y sus padres, para lograr una comprensión más integra de las complejidades involucradas en la deserción o adherencia a terapia en los casos de ASI (O'Keeffe et al., 2019).

Otra limitación de esta investigación es el restringido tamaño de la muestra y su escasa diversidad, que pudiera incidir en que algunas aristas del fenómeno no fueron posibles de observar. Por otro lado, dado el enfoque cualitativo utilizado, se describen similitudes y diferencias entre los casos de deserción y adherencia, no obstante, no es posible realizar comparaciones estadísticas entre los dos grupos (de adherencia y deserción). Otros elementos interesantes para profundizar en futuros estudios se relacionan con la edad, experiencia y enfoque clínico de los psicoterapeutas. También es importante considerar que, posiblemente, los terapeutas tengan más información de los casos de adherencia que de los de deserción, dada la mayor duración de la intervención y, por ende, la mayor estabilidad del vínculo psicoterapéutico. En ese sentido, la deserción implica tensión para todos los actores del proceso y es relevante mencionar procesos de frustración en los terapeutas, que pueden haber influido en su tendencia a destacar más los factores obstaculizadores del proceso en estos casos.

No obstante sus limitaciones, este estudio constituye un aporte novedoso y es pionero en Latinoamérica en el abordaje de la deserción y adherencia en adolescentes víctimas de ASI desde un enfoque cualitativo, que incorpora tanto elementos preterapéuticos como del proceso psicoterapéutico.

\section{Agradecimientos}

Este estudio se enmarca en un proyecto de investigación mayor que se denomina "Cambio psicoterapéutico en agresiones sexuales: perspectiva de los niños/as y adolescentes que han sido víctimas, sus padres y sus psicoterapeutas", el cual contó con el financiamiento del Fondo Nacional de Desarrollo Científico y Tecnológico (FONDECYT) N¹1140049 de CONICYT del Gobierno de Chile. Fue realizado por un equipo del cual forman parte las autoras, con el liderazgo de la investigadora principal, Claudia Capella. Se agradece a todo el equipo de investigación que hizo posible este trabajo y, especialmente, a los participantes de la investigación por compartir sus experiencias.

\section{Referencias}

Adam, J., Mrug, S., \& Knight, D. (2018). Characteristics of child physical and sexual abuse as predictors of psychopathology. Child abuse \& neglect, 86, 167-177.

Águila, D., Lama, X., Capella, C., Rodríguez, L., Dussert, D., Gutiérrez, C., \& Beiza, G. (2016). Narrativas de terapeutas sobre el proceso de cambio psicoterapéutico en niños, niñas y adolescentes que han sido víctimas de agresiones sexuales. Estudios de Psicología, 37(2-3), 344-364. https://doi.org/10.1080/02109395.201 $\underline{6.1204780}$

Altimir, C., Krause, M., De la Parra, G., Dagnino, P., Tomicic, A., Valdés, N., ... Vilches, 0. (2010) Clients', therapists', and observers' agreement on the amount, temporal location, and content of psychotherapeutic change and its relation to outcome. Psychotherapy Research, 20(4), 472-487.

Armbruster, P., \& Schwab-Stone, M. E. (1994). Sociodemographic characteristics of dropouts from child guidance clinic. Psychiatric Services, 45(8), 804-808. http:// ps.psychiatryonline.org/doi/abs/10.1176/ps.45.8.804 
Baruch, G., Vrouva, I., \& Fearon, P. (2009). A Follow? ? Young People that Dropout and Continue Psychotherapy: Service Implications for a Clinic in the Community. Child and Adolescent Mental Health, 14(2), 69-75. https://doi.org/10.1111/j.1475-3588.2008.00492.x

Bueno, A., Córdoba, J.A., Escolar, A., Carmona, J., \& Rodriguez, C (2001). El abandono terapéutico. Actas Esp Psiquiatría, 29(1), 33-40

Capella, C. (2017). Disequilibrium and Loss of Narrative Coherence in Identity Construction: A Piagetian Perspective on Trauma in Adolescent Victims of Sexual Abuse. Journal of Constructivist Psychology, 30(4), 323-338. https://doi.org/10.1080/107 $\underline{20537.2016 .1227737}$

Capella, C., Lama, X., Rodríguez, L., Águila, D., Beiza, G., Dussert, D., \& Gutierrez, C. (2016). Winning a race: narratives of healing and psychotherapy in children and adolescents who have been sexually abused. Journal of child sexual abuse, 25(1), 73-92. https://doi.org/10.1080/10538712.2015.1088915

Centro de Asistencia a Víctimas de Atentados Sexuales [CAVAS]. (2011). Centro de Asistencia a Víctimas de Atentados Sexuales: "Una Revisión de la experiencia". Instituto de Criminología (INSCRIM), Santiago, Chile.

Cornejo, M., \& Salas, N. (2011). Rigor y calidad metodológicos: un reto a la investigación social cualitativa. Psicoperspectivas, 10(2), 12-34. http://dx.doi.org/10.5027/psicoperspectivas-Vol10-Issue2-fulltext-144

Deakin, E., Gastaud, M., \& Tiellet, M. L. (2012). Child psychotherapy dropout: an empirical research review. Journal of Child Psychotherapy, 38(2), 199-209. https://doi. org/10.1080/0075417X.2012.684489

De Haan, A. M., Boon, A. E., de Jong, J. T., Hoeve, M., \& Vermeiren, R. R. (2013). A meta-analytic review on treatment dropout in child and adolescent outpatient mental health care. Clinical Psychology Review, 33(5), 698-711. Recuperado de http:// www.sciencedirect.com/science/article/pii/S0272735813000688

Demarest, K., Heger, A. H., Foy, D. W., \& Leskin, G. A. (1996). Factors associated with entry into therapy in children evaluated for sexual abuse. Child abuse \& neglect, 20(1), 63-68.

Di Matteo, M. R., \& DiNicola, D.D. (1982). Achieving Patient Compliance. The Psychology of the Medical Practitioner's Role. New York: Pergamon Press.

Dussert, D., Capella, C., Lama, X., Gutiérrez, C., Águila, D., Rodríguez, L., \& Beiza, G. (2017). Narrativas de Padres de Niños, Niñas y Adolescentes que Han Finalizado Psicoterapia por Agresiones Sexuales: Un Proceso de Superación Conjunta. Psykhe, 26(1), 1-14.

Edlund, M. J., Wang, P. S., Berglund, P. A., Katz, S. J., Lin, E., \& Kessler, R. C. (2002). Dropping out of mental health treatment: patterns and predictors among epidemiological survey respondents in the United States and Ontario. American Journal of Psychiatry, 159(5), 845-851. https://doi.org/10.1176/appi.ajp.159.5.845

Epperson, D. L., Bushway, D. J., \& Warman, R. E. (1983). Client self-terminations after one counseling session: Effects of problem recognition, counselor gender, and counselor experience. Journal of Counseling Psychology, 30(3), 307-315. https:// doi.org/10.1037/0022-0167.30.3.307

Eslinger, J., Sprang, G., \& Otis, M. (2014). Child and Caregiver Dropout in Child Psychotherapy for Trauma. Journal of Loss and Trauma, 19, 121-136. https://doi.org/10 $.1080 / 15325024.2012 .742720$

Fernández, O., Pérez, C., Gloger, S., \& Krause, M. (2015). Importancia de los Cambios Iniciales en la Psicoterapia con Adolescentes. Terapia psicológica, 33(3), 247-255.

Fondo de las Naciones Unidas para la Infancia [UNICEF], (2012). Cuarto estudio de maltrato infantil. Santiago: UNICEF. Recuperado de https://www.unicef.org/ spanish/publications/index 69639.html 
Freire, B. (1990). Estudio comparativo de tres grupos de pacientes con distinta continuidad terapéutica. Rev Asoc Esp Neuropsiquiatría, 10(33), 221-227.

Fuentes, S., \& Vásquez, V. (2017). Deserción Psicoterapéutica de Adolescentes que han sido Víctimas de Agresiones Sexuales: La Mirada de sus Psicoterapeutas. Memoria para optar al título de Psicóloga. Universidad de Chile.

Furnham, A., \& Wong, L. (2007). A cross-cultural comparison of British and Chinese beliefs about the causes, behaviour manifestations and treatment of schizophrenia. Psychiatry research, 151(1), 123-138.

Gearing, R. E., Schwalbe, C. S., \& Short, K. D. (2012). Adolescent adherence to psychosocial treatment: mental health clinicians' perspectives on barriers and promoters. Psychotherapy Research, 22(3), 317-326. https://doi.org/10.1080/10503307.201 1.653996

Guha, A., Luebbers, S., Papalia, N., \& Ogloff, J. (2019). A follow-up study of mental health service utilization in a cohort of 2433 sexually abused Australian children utilizing five years of medical data. Child abuse \& neglect, 90, 174-184.

Hamberger, L., Lohr, J., \& Gottlieb, M. (2000). Predictors of treatment dropout from a spouse abuse abatement program. Behavior Modification, 24(4), 528-552.

Hernández, J. L., Acosta F.J, \& Pereira J. (2009). Adherencia terapéutica. Cuadernos de psiquiatría comunitaria, 9 (1), 15-28.

Hetzel-Riggin, M. D., Brausch, A. M., \& Montgomery, B. S. (2007). A meta-analytic investigation of therapy modality outcomes for sexually abused children and adolescents: An exploratory study. Child abuse \& neglect, 31(2), 125-141.

Hillis, G., Eagles, A., \& Eagles, J. (1993). Premature termination of psychiatric contact. Int J Soc Psychiatry, 39(2), 100-107.

Horne, R., Weinman, J., Barber, N., Elliott, R., Morgan, M., Cribb, A., \& Kellar, I. (2005). Concordance, adherence and compliance in medicine taking. London: NCCSDO.

Jo, T., \& Almao, S. (2002). Abandono del tratamiento en una consulta externa de psiquiatría y psicología clínica. Revista Electrónica de portales médicos.com. Recuperado de http://www.portlesmedicos.com/monografías/abandono tratamiento/index.htm

Kazdin, A. E. (1996). Dropping out of child psychotherapy: Issues for research and implications for practice. Clinical Child Psychology and Psychiatry, 1(1), 133-156. https://doi.org/10.1177/1359104596011012

Kazdin, A., Mazurick, J., \& Bass, D. (1993). Risk for attrition in treatment of antisocial children and families. Journal of Clinical Child Psychology, 22(1), 2-16. https://doi. org/10.1207/s15374424jccp2201 1

Killaspy, H., Banerjee, S., King, M., \& Lloyd, M. (2000). Prospective controlled study of psychiatric out-patient non-attendance. The British Journal of Psychiatry, 176(2), 160-165. https://doi.org/10.1192/bjp.176.2.160

Lippert, T., Favre, T., Alexander, C., \& Cross, T. P. (2008). Families who begin versus decline therapy for children who are sexually abused. Child abuse \& neglect, 32(9), 859-868.

Luk, E. S., Staiger, P. K., Mathai, J., Wong, L., Birleson, P., \& Adler, R. (2001). Children with persistent conduct problems who dropout of treatment. European Child \& Adolescent Psychiatry, 10(1), 28-36. https://doi.org/10.1007/s007870170044

Marks, I., Lovell, K., Noshirvani, H., Livanou, M., \& Thrasher, S. (1998). Treatment of posttraumatic stress disorder by exposure and/or cognitive restructuring: $A$ controlled study. Archives of General Psychiatry, 55(4), 317-325. https://doi. org/10.1001/archpsyc.55.4.317

Matas, M., Staley, D., \& Griffin, W. (1992). A profile of the noncompliant patient: a thirty month review of outpatient psychiatry referrals. Gen Hosp Psychiat, 14(2), 124-130. 
McPherson, P., Scribano, P., \& Stevens, J. (2012). Barriers to Successful Treatment Completion in Child Sexual Abuse Survivors. Journal of interpersonal violence, 27 (1), 23-39. https://doi.org/10.1177/0886260511416466

Mendoza, M. (2016). Relación terapéutica y cambio psicoterapéutico, desde la experiencia subjetiva de adolescentes y sus terapeutas. (Tesis de Magíster, Departamento de Psicología, Universidad de Chile).

Núñez, L., Fuentes, S., Vásquez, V. \& Capella, C. (2016). Adolescentes víctimas de agresión sexual que desertan de la psicoterapia: La mirada de sus terapeutas. Ponencia presentada en el XII Congreso Latinoamericano de Investigación en Psicoterapia. Porto Alegre, Brasil. 20 al 22 de Octubre 2016.

Nock, M. K., \& Ferriter, C. (2005). Parent Management of Attendance and Adherence in Child and Adolescent Therapy: A Conceptual and Empirical Review. Clinical Child and Family Psychology Review, 8(2), 149-166. https://doi.org/10.1007/s10567005-4753-0

O'Keeffe, S., Martin, P., Goodyer, I., Wilkinson, P., Impact Consortium, \& Midgley, N. (2018) Predicting dropout in adolescents receiving therapy for depression. Psychotherapy Research, 28(5), 708-721. https://doi.org/10.1080/10503307.2017.1 393576

O'Keeffe, S., Martin, P., Target, M., \& Midgley, N. (2019). 'I Just Stopped Going': A Mixed Methods Investigation into Types of Therapy Dropout in Adolescents with Depression. Frontiers in Psychology, 10(75). https://doi.org/10.3389/ fpsyg.2019.00075

Ormhaug, S., \& Jensen, T. (2018). Investigating treatment characteristics and first-session relationship variables as predictors of dropout in the treatment of traumatized youth. Psychotherapy Research, 28(2), 235-249, https://doi.org/10.1 $\underline{080 / 10503307.2016 .1189617}$

Pelkonen, M., Marittunen, M., Laippala, P., \& Lönnqvist, J. (2000). Factors associated with early dropout from adolescent psychiatric outpatient treatment. Journal of American Academy of Child and Adolescent Psychiatry, 39(3), 329-36.

Pellerin, K. A., Costa, N. M., Weems, C. F., \& Dalton, R. F. (2010). An examination of treatment completers and non-completers at a child and adolescent community mental health clinic. Community mental health journal, 46(3), 273-281. https:// doi.org/10.1007/s10597-009-9285-5

Pereda, N., Guilera, G., Forns, M., \& Gómez-Benito, J. (2009). The international epidemiology of child sexual abuse: A continuation of Finkelhor (1994). Child abuse \& neglect, 33(6), 331-342.

Reich, C., \& Berman, J. (2018). Are psychotherapies with more dropouts less effective? Psychotherapy Research, 30(1), 23-40. https://doi.org/10.1080/10503307.2 018.1534018

Reis, B. F., \& Brown, L. G. (1999). Reducing psychotherapy dropouts: Maximizing perspective convergence in the psychotherapy dyad. Psychotherapy: Theory, Research, Practice, Training, 36(2), 123.

Riessman, C. (2008). Narratives Methods for the human sciences. California: Sage Publications.

Rondón, A.P., Otálora, I.L., \& Salamanca, Y. (2009). Factores que influyen en la deserción terapéutica de los consultantes de un centro universitario de atención psicológica. International Journal of Psychological Research, 2(2), 137-147.

Ross, J., \& Werbart, A. (2013) Therapist and relationship factors influencing dropout from individual psychotherapy: A literature review. Psychotherapy Research, 23(4), 394-418. https://doi.org/10.1080/10503307.2013.775528 
Saxe, G., Ellis, H., Fogler, J., \& Navalta, C. (2012). Innovations in Practice: Preliminary evidence for effective family engagement in treatment for child traumatic stresstrauma systems therapy approach to preventing dropout. Child and Adolescent Mental Health, 17(1), 58-61. https://doi.org/10.1111/j.1475-3588.2011.00626.x

Sepúlveda, M.G. (2013). Psicoterapia evolutiva con niños y adolescentes. Santiago, Chile: Mediterráneo.

Servicio Nacional de Menores (SENAME). (2015). Bases técnicas específicas: Programa de protección especializado modalidad maltrato infantil grave y abuso sexual. Recuperado de http://www.sename.cl/wsename/otros/proteccion/BTE.pdf

Swift, J., Spencer, J., \& Goode, J. (2018) Improving psychotherapy effectiveness by addressing the problem of premature termination: Introduction to a special section. Psychotherapy Research, 28,(5), 669-671. https://doi.org/10.1080/1050330 7.2018.1439192

Valles, M. (2003). Técnicas cualitativas de investigación social. Madrid: Ed. Síntesis.

Viale-Val, G., Rosenthal, R. H., Curtiss, G., \& Marohn, R. C. (1984). Dropout from adolescent psychotherapy: A preliminary study. Journal of the American Academy of Child Psychiatry, 23(5), 562-568.

Wamser-Nanney, R., \& Steinzor, C. (2017). Factors related to attrition from trauma-focused cognitive behavioral therapy. Child Abuse \& Neglect, 66, 73-83. http://dx. doi.org/10.1016/i.chiabu.2016.11.031

Wierzbicki, M., \& Pekarik, G. (1993). A meta-analysis of psychotherapy dropout. Professional Psychology: Research and Practice, 24(2), 190-195. https://doi. org/10.1037/0735-7028.24.2.190 